Abbreviated Key Title: Sch J Med Case Rep

ISSN 2347-9507 (Print) | ISSN 2347-6559 (Online)

Journal homepage: https://saspublishers.com

\title{
An Unusual Case of Colo-Vesical Fistula
}

Dr. Ravi Kumar", Dr. Uma Pradhan, Dr. P. N. Agarwal, Dr. G. J. Singh, Dr. Inder Verma

Faculty of Medical and Health Sciences, S.G.T. University, Gurgaon-Badli Road Chandu, Budhera, Gurugram, Haryana 122505, India

DOI: $10.36347 /$ simcr.2020.v08i11.011

| Received: 08.11.2020 | Accepted: 19.11.2020 | Published: 20.11.2020

*Corresponding author: Dr. Ravi Kumar

Abstract

Case Report

Mucinous colorectal adenocarcinoma is a subtype of colorectal cancer (CRC). It is characterized by the presence of abundant extracellular mucin. This mucin accounts for at least $50 \%$ of the tumor volume. Incidence of Mucinous colorectal adenocarcinoma is $10 \%-20 \%$ of CRC patients and it occurs more commonly in young females. This case report describes a case of colovesical fistula due to mucinous adenocarcinoma of sigmoid colon.

Keywords: Colovesical fistula, mucinous adenocarcinoma.

Copyright $\odot 2020$ The Author(s): This is an open-access article distributed under the terms of the Creative Commons Attribution 4.0 International License (CC BY-NC 4.0) which permits unrestricted use, distribution, and reproduction in any medium for non-commercial use provided the original author and source are credited.

\section{INTRODUCTION}

Colovesical fistula was first described by Rufus of Epheus in AD 200, though the first monograph on the case was produced by Cripps in 1888 [1]. It represents an abnormal communication between colon and the bladder. It is not a very common entity.

Majority etiological factors are- diverticular disease $(56.3 \%)$, carcinoma of colon $(20.1 \%)$, Crohn's disease $(9.1 \%)$, iatrogenic $(3.2 \%)$, radiotherapy $(3 \%)$, carcinoma of the cervix, carcinoma urinary bladder and appendicitis [2]. There are case reports of colovesical fistula following perforation of ingested foreign body [3].

\section{CASE}

23-year-old married female presented to SGT hospital surgical OPD with the complaints of passing debris in urine, yellowish discoloration and fowl smelling urine for last 10 days.

History of significant weight loss (approx. 10 $\mathrm{kg}$ in 6 months) was present. On General clinical examination patient was anaemic \& poorly nourished. Patient was hemodynamically stable. Local examination reveals left iliac fossa tenderness. All routine blood tests (LFT, KFT) were normal.

Ultrasonography reveals mass in the sigmoid colon abutting bladder. No lymphadenopathy. Normal liver and other viscera.

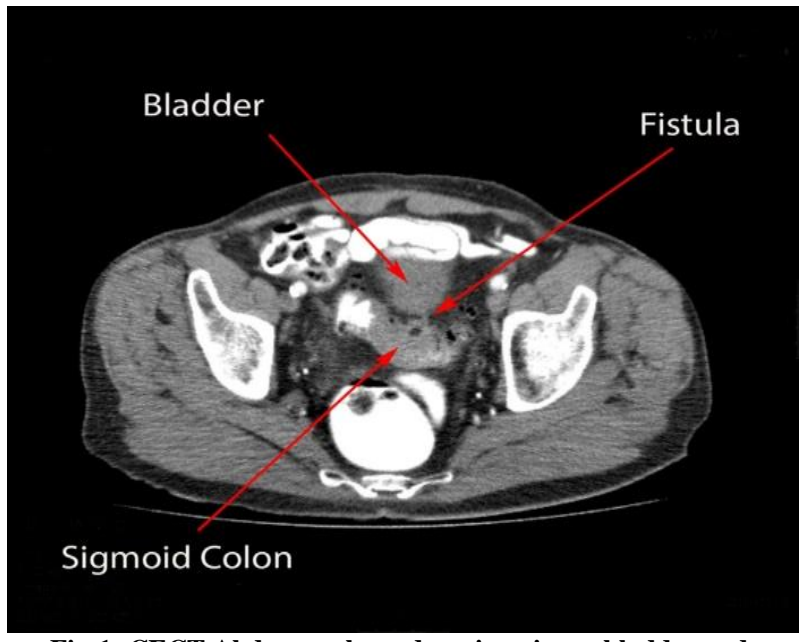

Fig-1: CECT Abdomen showed gas in urinary bladder and communication between bladder and sigmoid colon

CECT showed diffuse circumferential thickening of sigmoid colon. Polypoidal intraluminal mass extending into bladder, no hepatic metastasis (Fig$1)$.

Diagnostic cystoscopy was done which showed opening and a mass on the roof of urinary bladder. The fluid flushed through the bladder was coming out through anus. Biopsy was taken from the mass (Fig-2). 


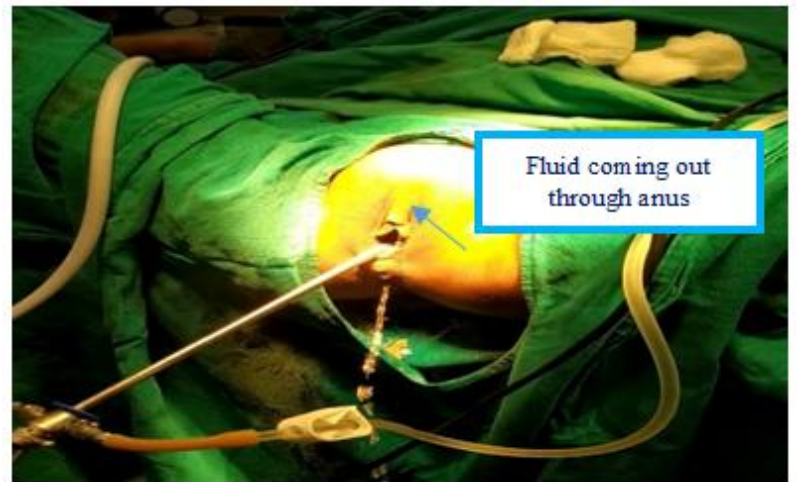

Fig-2: Image showing fluid coming out of anus during cystoscopy

Histopathology report shows mucinous adenocarcinoma colon infiltrating bladder wall (Fig-3).

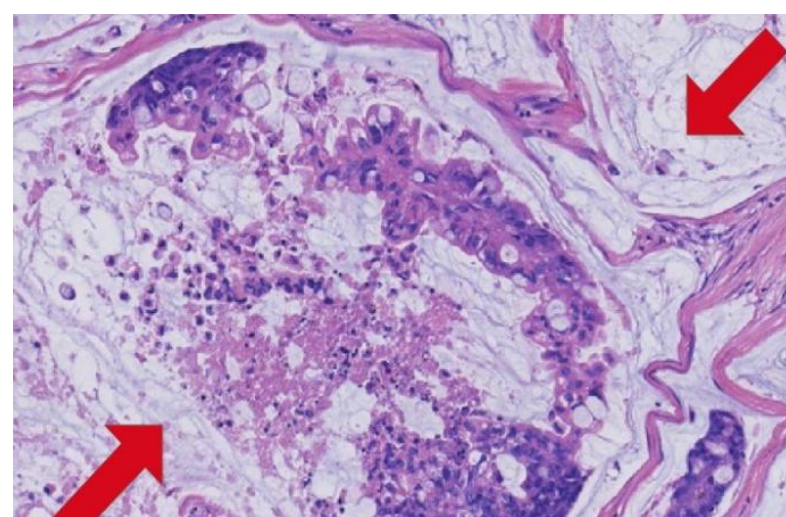

Fig-3: H\&E stained tissue section from a 23-year-old

female patient diagnosed with mucinous colorectal adenocarcinoma showing abundant extracellular mucin (red arrows) within the tumor complex

laparotomy.

Patient was taken up for exploratory

Intra operative findings - Colovesical fistula between the bladder and the sigmoid colon. Sigmoid colectomy with Partial cystectomy \& colo-rectal anastomosis and closure of bladder with suprapubic cystostomy was done.

Histo-pathology report confirmed mucinous adenocarcinoma. Resected specimen had negative margins and no lympho- vascular infiltration.

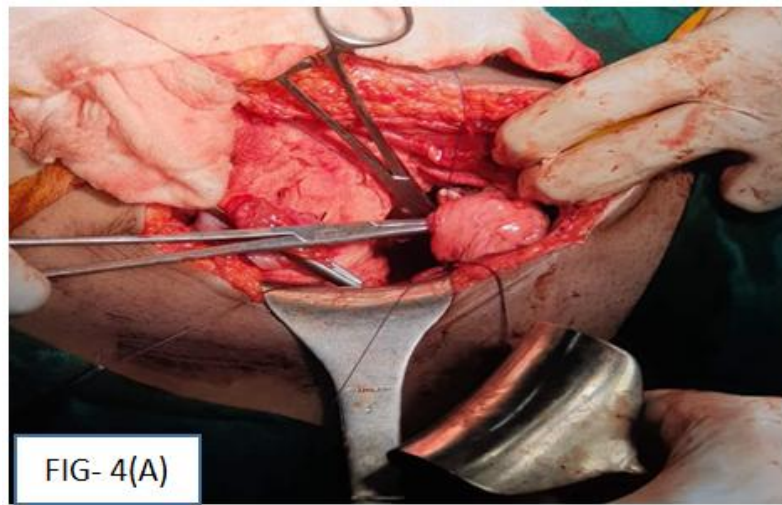

Fig-4(A): Intra- operative image showing communication between bladder and bowel

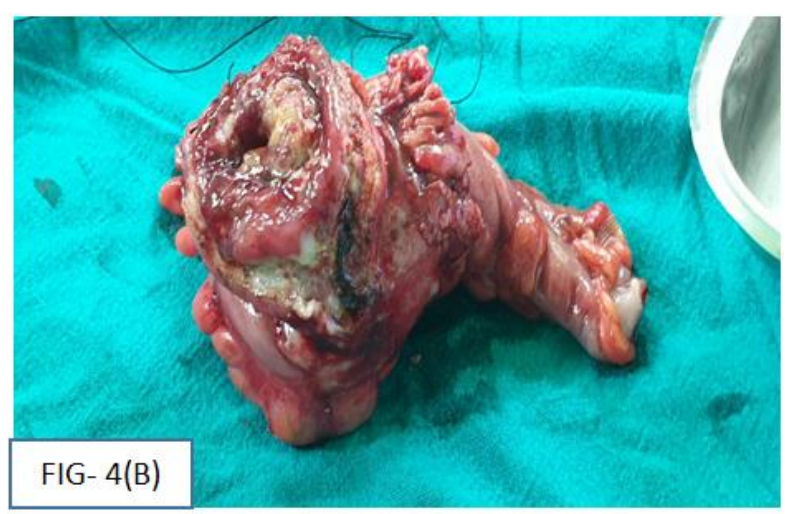

Fig-4(B): Resected sigmoid colon and fistula tract communicating with dome of bladder

Post-operative stay was uneventful and patient was discharged on $7^{\text {th }}$ day post-operative. She was advised for adjuvant therapy.

\section{DISCUSSION}

Colovesical fistula is an abnormal communication between the colon and urinary bladder. Various aetiologies of these fistulas include inflammatory bowel disease, advanced intestinal or urinary malignancies, radiotherapy, pelvic surgery, trauma or iatrogenic injuries [2, 4-6]. Depending on the bowel segment involved, enterovesical fistula can be divided into ileo-vesical, appendico-vesical, colovesical or recto-vesical $[5,6]$. Colovesical fistula is commonly seen in the setting of diverticular disease or colon cancer [4] while rectovesical fistula is usually due to trauma or neoplasm $[4,5,7]$.

Clinical manifestations of colovesical fistula include fecaluria, pneumaturia, recurrent UTIs, abdominal pain and malodourous urine [2]. Diagnostic workup includes cystography, barium studies, ultrasonography, CT scan. 
Trans-abdominal ultrasonography shows floating intra-luminal debris in the urinary bladder and abnormal approximation of a bowel loop and urinary bladder with indecipherable intervening wall separation. Trans-rectal and trans-vaginal ultrasonography can be done as they increase the chances of detection of fistula. However, it is highly operator dependent.

Plain abdominal X-ray and barium studies are usually inconclusive [2].

Cystography can give direct visualization of the fistulous tract or an indirect evidence by contrast opacification of bowel loops. Cross-sectional imaging (CT/ MRI) is the modality of choice as it can demonstrate the location and etiology of fistulous tract and also the extent of underlying disease ${ }^{2,8}$. Findings suggestive of a communication between bowel and urinary bladder are presence of air within the bladder (when there is no prior history of bladder instrumentation or active urinary tract infection with a gas-forming organism), oral contrast medium within the bladder (in non IV contrast-enhanced scans) or focal eccentric bowel wall thickening [8]. Colonoscopy can be done in cases of colo-vesical fistula to assess underlying colonic pathology with tissue sampling.

Management is mainly surgical depending on the presentation of the patient and underlying pathology. Pre -operative treatment includes bowel rest, total parenteral nutrition and antibiotics for UTIs and treatment of the underlying disease.

\section{CONCLUSION}

Very few studies have been done in colovesical fistula incidence among Indian population.
Colonoscopy and cystoscopy form an important part of the investigations. Single stage resection of the diseased bowel with anastomosis and primary closure of bladder defect remains the mainstay of the treatment. Laparoscopic resections are increasing in popularity and deliver encouraging results comparable to open resection.

\section{REFERENCE}

1. Cripps H. Passage of air and faeces from urethra. Lancet, 1888; 2: 619.

2. Golabek T, Szymanska A, Szopinski T, Bukowczan J, Furmanek M, Powroznik J, Chlosta P. Enterovesical fistulae: aetiology, imaging, and management. Gastroenterology research and practice. 2013 Oct;2013.

3. Karamchandani MC, West CF Jr. Vesicoenteric fistulas. Am J Surg, 1984;147:681-83.

4. Kavanagh D, Neary P, Dodd JD, Sheahan KM, O'Donoghue D, Hyland JM. Diagnosis and treatment of enterovesical fistulae. Colorectal Dis. 2005;7:286-91.

5. Chen WS, Hsieh JH, Jiang JK, Lin JK, Hsu H, Lin TC. Enterovesical fistula: 10 years of experience. Chin Med J, 1997;59:283-88.

6. Gross M, Peng B. Appendico-vesical fistula. J Urol.1969;102:697-98.

7. Li S, Chen Z, Zhang Q, Huang C, Wang Z, Du S. Four cases of enterovesical fistula and the importance of $\mathrm{CT}$ in the diagnosis. BJR| case reports. 2017 Jan 1:20150124.

8. Goldman SM, Fishman EK, Gatewood OMB. CT in the diagnosis of enterovesical fistulae. AJR Am J Roentgenol. 1985; 144:1229-33. 